\title{
A COUNTABLY PARACOMPACT NONNORMAL SPACE
}

\author{
W. WEISS ${ }^{1}$
}

\begin{abstract}
A countably paracompact, first countable, separable, submetrizable, locally compact space which is not normal is constructed.
\end{abstract}

The theorem of $M$. Wage [7] which states a relationship between normal nonmetrizable Moore spaces and countably paracompact nonnormal Moore spaces has led to a search for first countable nonnormal countably paracompact spaces. Three examples have been constructed: one by Wage [6] which is separable submetrizable, one by van Douwen [2] which is separable and locally compact, and one by Vaughan [5] which is locally compact and even countably compact.

We now construct a locally compact, first countable, nonnormal countably paracompact space, $\langle X, \tau\rangle$, which is both submetrizable and separable. Since $X$ is a subset of the real line and $\tau$ is finer than the Euclidean topology, by a theorem of $[3],\langle X, \tau\rangle$ is also real compact.

The construction of $\langle X, \tau\rangle$. Let $\mathbf{Q}$ be the rationals, $\mathbf{R}$ the reals and $c=|\mathbf{R}|$. We will define a topology $\tau$ on a subset $X<\mathbf{R}$ by inductively defining subsets $A_{\beta}=\left\{a_{\alpha}: \alpha<\beta\right\}$ and $B_{\beta}=\left\{b_{\alpha}: \alpha<\beta\right\}$ of $\mathbf{R}$ and topologies $\tau_{\beta}$ on $X_{\beta}=A_{\beta} \cup B_{\beta}$ $\cup \mathbf{Q}$, using techniques developed in [3] which originated in [4]. When we are finished with the inductive construction we will let $A=A_{c}, B=B_{c}, X=A \cup B \cup$ $Q$ and $\tau=\tau_{c}$.

We use the following inductive hypothesis I. H. $(\beta)$ for $\beta<c$ :

(i) for all $\alpha<\beta, \mathscr{P}\left(X_{\alpha}\right) \cap \tau_{\beta}=\tau_{\alpha}$,

(ii) $\tau_{\beta}$ refines the Euclidean topology on $X_{\beta}$,

(iii) $\mathbf{Q}$ is dense in $\left\langle X_{\beta}, \tau_{\beta}\right\rangle$,

(iv) $\left\langle X_{\beta}, \tau_{\beta}\right\rangle$ is locally compact,

(v) $\left\langle X_{\beta}, \tau_{\beta}\right\rangle$ is locally countable (i.e. each point has a countable neighbourhood),

(vi) $A_{\beta}$ and $B_{\beta}$ are closed in $\left\langle X_{\beta}, \tau_{\beta}\right\rangle$.

To begin, we let $A_{0}=B_{0}=\varnothing$ and $\tau_{0}$ be the discrete topology on $\mathbf{Q}$. At limit stage $\lambda$, for $\lambda<c$, we let $A_{\lambda}=\bigcup\left\{A_{\alpha}: \alpha<\lambda\right\}, B_{\lambda}=\bigcup\left\{B_{\alpha}: \alpha<\lambda\right\}$ and $\tau_{\lambda}$ be the topology on $X_{\lambda}$ generated by $\cup\left\{\tau_{\alpha}: \alpha<\lambda\right\}$. It is easy to check that the inductive hypotheses are fulfilled in these cases.

Before explaining the successor step we first let $\left\{\mathcal{C}_{\alpha}=\left\langle C_{\alpha}^{n}: n \in \omega\right\rangle: \alpha<c\right\}$ enumerate all countable sequences of countable subsets of $\mathbf{R}$ such that each sequence is repeated $c$ times. Also, let $\left\{E_{\alpha}: \alpha<c\right\}$ enumerate all countable subsets of $\mathbf{Q}$.

Received by the editors August 30, 1979.

AMS (MOS) subject classifications (1970). Primary 54D20, 54D30; Secondary 54A25.

${ }^{1}$ Research partially supported by NSTRC Grant No. 366011430. 
At stage $\beta+1$ consider $E_{\beta}$ and $\bigcup_{\beta}$. Let $F_{\beta}=\{x \in \mathbf{R}$ : for all Euclidean open neighbourhoods $N$ of $x$, the set $\left\{n: N \cap C_{\beta}^{n} \neq \varnothing\right\}$ is infinite $\}$. We divide into two cases.

In the first case we suppose that $\cup\left\{C_{\beta}^{n}: n \in \omega\right\} \subseteq X_{\beta}$ and $\left|F_{\beta}\right|=c$. We pick $x \in F \backslash X_{\beta}$. There is a sequence $\left\{c_{n_{k}}: k \in \omega\right\}$ converging to $x$ in the Euclidean topology such that each $c_{n_{k}} \in C_{\beta}^{n_{k}}$ and either the sequence is a subset of $A_{\beta} \cup \mathbf{Q}$ or $B_{\beta} \cup \mathbf{Q}$. Without loss of generality we assume $\left\{c_{n_{k}}: n \in \omega\right\} \subseteq A_{\beta} \cup \mathbf{Q}$. We now spilt into two subcases.

In the first subcase we suppose that $x$ is a Euclidean limit point of $E_{\beta}$ and that $E_{\beta}$ is somewhere dense in the Euclidean topology. Pick $b \in \mathbf{R} \backslash\left(X_{\beta} \cup\{x\}\right)$ such that $b$ is a Euclidean limit point of $E_{\beta}$. Choose $\left\{y_{k}: k \in \omega\right\}$ and $\left\{z_{k}: k \in \omega\right\}$ disjoint subsets of $E_{\beta}$ with $\left\{z_{k}: k \in \omega\right\} \cap\left\{c_{n_{k}}: k \in \omega\right\}=\varnothing$ such that $\left\{y_{k}: k \in \omega\right\}$ converges to $x$ and $\left\{z_{k}: k \in \omega\right\}$ converges to $b$ in the Euclidean topology. Now pick compact, clopen, countable neighbourhoods $U_{k}$ of $c_{n_{k}}$ such that $\left\{U_{k}: k \in \omega\right\}$ is discrete in $\tau_{\beta}$ with the diameter of $U_{k}<1 / k$. Also ensure that for each $k \in \omega$, $U_{k} \cap\left(B_{\beta} \cup\left\{z_{i}: i \in \omega\right\}\right)=\varnothing$. Now let $V_{m}^{x}=\cup\left\{U_{k}: k \geqslant m\right\} \cup\left\{y_{k}: k \geqslant m\right\}$ and $V_{m}^{b}=\left\{z_{k}: k>m\right\}$. Let $A_{\beta+1}=A_{\beta} \cup\{x\}$ and $B_{\beta+1}=B_{\beta} \cup\{b\}$ and $\tau_{\beta+1}$ be the topology generated by $\tau_{\beta} \cup\left\{V_{m}^{x}: m \in \omega\right\} \cup\left\{V_{m}^{b}: m \in \omega\right\}$.

For the second subcase, we assume that the first subcase does not happen. Hence $x$ is a Euclidean limit point of $\mathbf{Q} \backslash E_{\beta}$ and $\mathbf{Q} \backslash E_{\beta}$ is somewhere dense. We now proceed in a manner similar to the first subcase, replacing $E_{\beta}$ by $Q \backslash E_{\beta}$.

For the second case, in which $\cup\left\{C_{\beta}^{n}: n \in \omega\right\} \mathbb{Z} X_{\beta}$ or $\left|F_{\beta}\right|<c$, we simply proceed as in the first case, ignoring all reference to the set $F_{\beta}$. Note that in any case either $E_{\beta}$ has limit points in both $A_{\beta+1}$ and $B_{\beta+1}$ or $\mathbf{Q} \backslash E_{\beta}$ has limit points in both $A_{\beta+1}$ and $B_{\beta+1}$. We are now finished with the inductive construction.

The properties of $\langle X, \tau\rangle$. It is straightforward to check that $\langle X, \tau\rangle$ is locally compact, locally countable, separable and submetrizable. $A$ and $B$ are closed disjoint subsets of $X$ which cannot be separated by open sets, since if $U \in \tau$, then for some $\beta, U \cap \mathbf{Q}=E_{\beta}$ and $X \backslash \bar{U}=\mathbf{Q} \backslash E_{\beta}$ at least one of which has limit points in both $A$ and $B$.

To show that $\langle X, \tau\rangle$ is countably paracompact, it suffices to show that any countable open cover $\left\{U_{n}: n \in \omega\right\}$ with each $U_{n} \subseteq U_{n+1}$ has a locally finite refinement. Let $G_{0}=U_{0}$ and $G_{n+1}=U_{n+1} \backslash U_{n}$. Let $\Phi=\{x \in X$ : for all Euclidean open neighbourhoods $N$ of $x$, the set $\left\{n \in \omega: G_{n} \cap N \neq \varnothing\right\}$ is infinite $\}$.

We claim that $|\Phi|<\aleph_{0}$. Suppose not. Let $D$ be a countable subset of $\Phi$ which is somewhere dense for the Euclidean topology. For each $d \in D$ and for each $n \in \omega$ pick $g_{n}^{d} \in G_{n}$ such that in the $\tau$ topology $d$ is in the closure of $\left\{g_{n}^{d}: n \in \omega\right\}$. Let $C^{n}=\left\{g_{n}^{d}: d \in D\right\}$ and $C=\left\{C^{n}: n \in \omega\right\}$

Let $\Psi=\{x \in \mathbf{R}$ : for each Euclidean open neighbourhood $N$ of $x$ the set $\left\{n \in \omega: N \cap C^{n} \neq \varnothing\right\}$ is infinite $\}$. Now $\Psi$ is closed in the Euclidean topology, and contains $D$. Therefore $\Phi \subseteq \Psi$ and hence $\Psi$ has cardinality $c$.

Now, there is some $\beta<c$ such that $C \leqslant X_{\beta}$ and $\left\langle C^{n}: n \in \omega\right\rangle=\mathcal{C}_{\beta}$ and hence by construction without loss of generality we can assume there is some $a \in A \cap \Psi$ (since otherwise there is $b \in B \cap \Psi$ ). Furthermore every $\tau$ neighbourhood of $a$ 
intersects infinitely many $C^{n}$ 's and hence infinitely many $G_{n}$ 's and therefore $a$ is not in the $\tau$ interior of any $U_{n}$. This contradiction shows $|\Phi|<\aleph_{0}$.

Let $Y=X-\Phi .\left\{G_{n}: n \in \omega\right\}$ is locally finite for the Euclidean topology on $Y$. Hence there are Euclidean open sets $V_{n} \supseteq G_{n}$ such that $\left\{V_{n}: n \in \omega\right\}$ is locally finite for the Euclidean topology on $Y$.

Enumerate $\Phi$ as $\left\{x_{n}: n \in \omega\right\}$ and for each $n$ pick a compact, countable $O_{n} \in \tau$ such that $x_{n} \in O_{n}$, diameter of $O_{n}<1 / n$ and $O_{n}<U_{k}$ for some $k$. Let $\Omega=$ $\cup\left\{O_{n}: n \in \omega\right\} . \Omega$ is a clopen metrizable subset of $\langle X, \tau\rangle$.

Now, let $\left\{O_{n}^{*}: n \in \omega\right\}$ be a locally finite refinement of $\left\{O_{n}: n \in \omega\right\}$. Let $V_{n}^{*}=\left(V_{n} \backslash \Omega\right) \cap U_{n}$ for each $n \in \omega$. It is straightforward to check that $\left\{V_{n}^{*}\right.$ : $n \in \omega\} \cup\left\{O_{n}^{*}: n \in \omega\right\}$ refines $\left\{U_{n}: n \in \omega\right\}$.

REMARK. $\langle X, \tau\rangle$ is not paracompact, since it is not normal. In fact there is an open cover of cardinality $\aleph_{1}$ which has no point-countable refinement. Note that since $\langle X, \tau\rangle$ is separable any point-countable cover is countable.

Let $\left\{x_{\alpha}: \alpha \in c\right\}$ be an enumeration of $X$ such that each initial segment is open and $\mathbf{Q}=\left\{x_{n}: n \in \omega\right\}$. For each $x_{\beta}$ find a countable neighbourhood $U_{\beta}$ of $x_{\beta}$ contained in $\left\{x_{\alpha}: \alpha<\beta\right\}$. Define a function $f: X \rightarrow \omega_{1}$ as follows:

$$
f\left(x_{\alpha}\right)=\left\{\begin{array}{l}
\alpha \quad \text { if } \alpha \in \omega_{1}, \\
\sup \left\{f(y): y \in U_{\alpha}\right\} \quad \text { if } \alpha \notin \omega_{1} .
\end{array}\right.
$$

Let $V_{\alpha}=\{x \in X: f(x)<\alpha\} .\left\{V_{\alpha}: \alpha \in \omega_{1}\right\}$ has no countable subcover.

Modification. We can modify the construction of $\langle X, \tau\rangle$ to obtain essentially the same space constructed in [1]. We eliminate all references to the sets $A_{\beta}$ and $B_{\beta}$.

Let $\left\{\mathscr{P}_{\alpha}=\left\langle P_{\alpha}^{1}, P_{\alpha}^{2}\right\rangle: \alpha \in c\right\}$ enumerate all.pairs of countable subsets of $\mathbf{R}$ such that each pair is repeated $c$ times. We let our new inductive hypothesis be parts (i) - (v) of the previous inductive hypothesis.

The construction at limit stages is the same as before. At successor stage $\beta+1$ we add a point $x_{\beta+1}$ ensuring that if $P_{\beta}^{1}$ and $P_{\beta}^{2}$ have $c$ Euclidean limit points in common then $x_{\beta+1}$ is a limit point of both $P_{\beta}^{1}$ and $P_{\beta}^{2}$ in the new topology.

When we finish the construction the new space will have the property that if $H$ and $K$ are subsets of $X$ and if $H$ and $K$ have uncountably many Euclidean limit points in common then $H$ and $K$ have $c$ limit points in common in the new topology. We can use this property to show that the new space is normal. The proof is not dissimilar to the previous proof that $\langle X, \tau\rangle$ was countably paracompact and to the proof in [1]. Thus the new space will be separable, normal locally compact but with a cover of cardinality $\aleph_{1}$ having no point-countable refinement.

\section{REFERENCES}

1. E. van Douwen, A technique for constructing honest locally compact submetrizable examples, preprint. 2. 1 (1976), 239-242. , Hausdorff gaps and a nice countably paracompact non-normal space, Topology Proceedings

3. I. Juhasz, K. Kunen and M. E. Rudin, Two more hereditarily separable non-Lindelof spaces, Canad. J. Math. 28 (1976), 998-1005. 
W. WEISS

4. A. Ostaszewaki, On countably compact, perfectly normal spaces, J. London Math. Soc. 14 (1976), S05-516.

5. J. Vaughan, A countably compact, first countable, non-normal $T_{2}$-space, Proc. Amer. Math. Soc. 75 (1979), 339-342.

6. M. Wage, Non-normal spaces, set-theoretic topolozy, G. M. Reed, ed., Academic Preas, New York, 1976.

7. M. Wage, W. Fleissener and G. M. Reed, Normality versus countable paracompactmess in perfect spaces, Bull. Amer. Math. Soc. 82 (1970), 635-639.

Departmant of Matheachtics, University of Toronto, ernndale Collbob, Mmssissauga, Ontardo, LSL 1C6, Canada 